\title{
Genetic contribution to radiographic severity in osteoarthritis of the knee
}

\author{
Ana M Valdes, ${ }^{1}$ Sally Doherty, ${ }^{2}$ Kenneth R Muir, ${ }^{3}$ Weiya Zhang, ${ }_{1}^{4}$ Rose A Maciewicz, ${ }^{5}$ \\ Margaret Wheeler, ${ }^{2}$ Nigel Arden, ${ }^{6}$ Cyrus Cooper, ${ }^{7}$ M Doherty ${ }^{2}$
}

\begin{abstract}
- Additional material is published online only. To view this file please visit the journal online (http://ard.bmj.com/ content/early/recent).
\end{abstract}

${ }^{1}$ Twin Research Unit, Kings College London, London, UK ${ }^{2}$ Academic Rheumatology, University of Nottingham, Nottingham, UK

${ }^{3}$ Health Sciences Research Institute, University of Warwick, Warwick, UK

${ }^{4}$ Academic Rheumatology, Nottingham University,

Nottingham, UK

${ }^{5}$ Respiratory \& Inflammation

iMed, AstraZeneca,

Loughborough, UK

${ }^{6}$ University of Southampton,

Southampton, UK

${ }^{7}$ MRC Epidemiology Resource

Centre, University of

Southampton, Southampton, UK

\section{Correspondence to}

Dr Ana M Valdes, Kings College London, Twin Research Unit,

St Thomas Hospital, Lambeth Palace Rd, London SE1 7EH, UK; ana.valdes@kcl.ac.uk

Accepted 19 April 2012 Published Online First 25 May 2012

\begin{abstract}
Objective Knee osteoarthritis $(\mathrm{OA})$ has a significant genetic component. The authors have assessed the role of three variants reported to influence risk of knee $O A$ with $p<5 \times 10^{-8}$ in determining patellofemoral and tibiofemoral Kellgren Lawrence (K/L) grade in knee OA cases.
\end{abstract}

Methods 3474 knee OA cases with sky-line and weight-bearing antero-posterior $x$-rays of the knee were selected based on the presentation of $\mathrm{K} / \mathrm{L}$ grade $\geq 2$ at either the tibiofemoral or patellofemoral compartments for one or both knees. Patients belonging to three UK cohorts, were genotyped for rs 143383, rs4730250 and rs 11842874 mapping to the GDF5, COG5 and MCF2L genes, respectively. The association between tibiofemoral $\mathrm{K} / \mathrm{L}$ grade and patellofemoral $\mathrm{K} / \mathrm{L}$ grade was assessed after adjusting for age, gender and body mass index.

Results No significant association was found between the rs4730250 and radiographic severity. The rs11842874 mapping to MCF2L was found to be nominally significantly associated with patellofemoral $\mathrm{K} / \mathrm{L}$ grade as a quantitative trait $(p=0.027)$ but not as a binary trait. The GDF5 single nucleotide polymorphism rs143383 was associated with tibiofemoral K/L grade $(\beta=0.05$ (95\% Cl 0.02 to 0.08) $p=0.0011)$.

Conclusions Our data indicate that within individuals affected by radiographic knee OA, OAGDF5 has a modest but significant effect on radiographic severity after adjustment for the major risk factors.

\section{INTRODUCTION}

Osteoarthritis $(\mathrm{OA})$ is the most common joint disorder ${ }^{1}$ and has an important genetic component associated with its development. ${ }^{2}$ Many genes contribute modestly to the risk for developing OA. ${ }^{23}$ To date, variants in three genes have been reproducibly associated in European-descent populations with knee $O A$ with $p<5 \times 10^{-8}$. These are: a variant in the promoter region of the growth differentiation factor 5 (GDF5) gene, ${ }^{4}$ a variant mapping to a gene cluster in chromosome 7 including the component of oligomeric golgi complex 5 (COG5) gene $\mathrm{s}^{5}$ and a variant in the MCF.2 cell-line-derived transforming sequence-like (MCF2L) gene. ${ }^{6}$ The role of GDF5 in the pathogenesis of OA has been widely explored ${ }^{7}$ but little is known about the biological processes influenced by the other two variants.

The most common definition of OA for genetic studies has been a radiographic one, based on the Kellgren-Lawrence (K/L) system ${ }^{8}$ using the cut-off value of 2 as a case definition, which corresponds to $\mathrm{x}$-ray-revealed presence of definite osteophytes and possible joint space narrowing. However, the presence of osteophytes is extremely common in the general population, ${ }^{9}$ and a $\mathrm{K} / \mathrm{L}$ grade $=2$ is not necessarily of clinical concern and, in fact, it rarely leads to total joint replacement. ${ }^{10-12}$ An important aspect to investigate is the extent to which genetic variation influences radiographic severity. In three independent study cohorts from the UK, we have investigated the role of the variants so far identified to be reproducibly associated with knee OA in Caucasians. We have assessed their role among individuals with radiographic evidence of knee OA to the extent of radiographic damage, as defined by the $\mathrm{K} / \mathrm{L}$ grade, at both the tibiofemoral and patellofemoral compartments.

\section{SUBJECTS AND METHODS Patients}

Knee OA patients, taken from two case-control cohorts, Genetics of Osteoarthritis and Lifestyle (GOAL) Study and the Nottingham Case-Control Study, were included. Patients recruited from the community selected from a population-based study, the Hertfordshire Cohort Study, were also included. A description of each cohort is detailed in the online supplementary methods.

\section{Genotyping}

Genotyping of the rs143383, rs4730250 and rs11842874 single nucleotide polymorphisms (SNPs) was carried out by Kbioscience Ltd, Hertfordshire, UK, using the KASPar chemistry. Further details on quality control in the genotyping are shown in the online supplementary methods.

\section{Statistical analysis}

The association between SNPs and radiographic severity of knee OA was first assessed by linear regression using as outcome variables, the $\mathrm{K} / \mathrm{L}$ grade at the tibiofemoral and patellofemoral compartments, and as independent variables the number of risk alleles (0,1 or 2) at each SNP. Age, sex and body mass index (BMI) were included as covariates. Mean $\mathrm{K} / \mathrm{L}$ values adjusted for covariates and cohort of origin for each genotype were derived from ANOVA.

Significant results were also tested using logistic regression analyses (adjusting for age, sex and BMI) comparing patients with $\mathrm{K} / \mathrm{L}=2$ with patients with $\mathrm{K} / \mathrm{L} \geq 3$. Meta-analyses of data from different cohorts were performed using R V.2.13.1 (http:// 
www.r-project.org/). Random effects models were not used as no heterogeneity was seen in any of the analyses. The statistical power to detect associations with severity of $\mathrm{OA}$, given the number of individuals sampled at an $\alpha$ level $=0.05$, was calculated separately for the two SNPs that showed nominal associations with severity of $\mathrm{OA}$ based on the risk allele frequency of each marker using a normal approximation, using the Quanto Software V.1.2.4

\section{RESULTS}

The descriptive characteristics of the study cohorts, including the distribution of $\mathrm{K} / \mathrm{L}$ grades at the tibiofemoral and patellofemoral grades, are presented in table 1 .

The individual association between the polymorphisms and the measures of severity of knee OA for each cohort adjusting for age, sex and BMI were computed, and the resulting coefficients were meta-analysed (table 2). Of the three variants, the risk allele at the GDF5 SNP is significantly associated with

Table 1 Descriptive statistics of knee OA cases in the study cohorts

\begin{tabular}{llll}
\hline Study cohort & Goal & HCS & Nottingham \\
\hline Patients collected from & Secondary care & Community & Secondary care \\
Number of knee OA cases & 1879 & 557 & 1038 \\
F\% & $48.6 \%$ & $45.6 \%$ & $57.0 \%$ \\
Age (SD) & $68.23(7.22)$ & $65.62(2.62)$ & $70.02(9.47)$ \\
Body mass index (SD) & $30.49(5.38)$ & $27.82(4.48)$ & $29.97(5.74)$ \\
Patellofemoral K/L grade & & & \\
$<2$ & $19.5 \%$ & $2.9 \%$ & $25.0 \%$ \\
2 & $34.6 \%$ & $43.1 \%$ & $46.8 \%$ \\
3 & $34.2 \%$ & $24.1 \%$ & $19.8 \%$ \\
4 & $11.7 \%$ & $30.0 \%$ & $8.4 \%$ \\
Tibiofemoral K/L grade & & & \\
$<2$ & $14.7 \%$ & $73.2 \%$ & $2.2 \%$ \\
2 & $16.8 \%$ & $18.9 \%$ & $9.3 \%$ \\
3 & $46.2 \%$ & $7.2 \%$ & $61.5 \%$ \\
4 & $22.2 \%$ & $0.7 \%$ & $27.0 \%$ \\
Risk allele frequencies & & & \\
rs143383 (T) & $65.81 \%$ & $61.67 \%$ & $64.40 \%$ \\
rs11842874 (A) & $93.82 \%$ & $92.84 \%$ & $94.61 \%$ \\
rs4730250 (T) & $15.63 \%$ & $15.57 \%$ & $17.51 \%$ \\
\hline
\end{tabular}

GOAL, Genetics of Osteoarthritis and Lifestyle study; HCS, Hertfordshire Cohort Study; Nottingham, Nottingham Case-Control Study. higher tibiofemoral K/L grade even after adjusting for the six tests carried out. The MCF2L variant was only nominally significantly associated with patellofemoral $\mathrm{K} / \mathrm{L}$ and is not significant if adjusted for multiple tests (table 2).

The test results with $\mathrm{K} / \mathrm{L}$ grade might be reflecting the already known association with risk of OA. We therefore performed a sub-analyses on tibiofemoral $\mathrm{K} / \mathrm{L}$ and GDF5 and patellofemoral $\mathrm{K} / \mathrm{L}$ and MCF2L only among patients affected the tibiofemoral and patellofemoral compartments, respectively (K/L > =2 at the relevant compartment). There is a clear linear relationship between the mean tibiofemoral K/L grade (adjusted for covariates) and the number of predisposing $T$ alleles carried at rs143383 (figure 1A). The difference observed between genotypes is 0.055 of $\mathrm{K} / \mathrm{L}$ grade, and the current study has a power of $69 \%$ to detect such difference with $p<0.05$. The rs 11842874 homozygote for the protective $G$ allele does not have a lower mean patellofemoral $\mathrm{K} / \mathrm{L}$ than the heterozygote carrier of the risk allele A. The average difference between genotypes is 0.027 of $\mathrm{K} / \mathrm{L}$ grade. The study has a power of $10 \%$ for such an effect size given the allele frequency (figure 1B). The forest plots illustrating the association coefficients and the overall meta-analysis summary statistic of the individual cohort, are also shown for this sub-analysis in figure $1 \mathrm{C}$ for rs 143383 and tibiofemoral K/L, achieving a $\mathrm{p}=0.0011$ by meta-analysis; in figure $1 \mathrm{D}$, we show the association between rs11842874 and patellofemoral K/L which achieves a $\mathrm{p}=0.027$ by meta-analysis.

$\mathrm{K} / \mathrm{L}$ grade is a discrete measure and it is not truly normally distributed. We therefore ran logistic regressions for the two nominally significant results. We assessed the GDF5 effect including knee $\mathrm{OA}$ cases with tibiofemoral $\mathrm{K} / \mathrm{L}=2$ versus $\mathrm{K} / \mathrm{L} \geq 3$ (figure 1E) and the MCF2L effect comparing patellofemoral K/ $\mathrm{L}=2$ versus $\mathrm{K} / \mathrm{L} \geq 3$ (figure $1 \mathrm{~F}$ ). The GDF5 result remains statistically significant ( $\mathrm{p}=0.008)$, but the association of MCF2L with patellofemoral grade is not statistically significant $(\mathrm{p}=0.08)$. Thus, the association between GDF5 and tibiofemoral grade is not a statistical artifact due to the non-normality of the distribution of $\mathrm{K} / \mathrm{L}$ grades.

\section{DISCUSSION}

We have shown that two of the three genes which have, to date, been convincingly implicated in genetic risk of knee OA also correlate with the extent of radiographic damage at the

Table 2 Individual study genetic association estimates with patellofemoral (PF) and tibiofemoral (TF) Kellgren-Lawrence (K/L) grade and meta-analysis summary statistics. All results are adjusted for age, sex and body mass index

\begin{tabular}{|c|c|c|c|c|c|c|c|c|}
\hline \multirow[b]{2}{*}{ Gene } & \multirow[b]{2}{*}{ Variant } & \multirow[b]{2}{*}{ Cohort } & \multicolumn{3}{|c|}{ PF K/L } & \multicolumn{3}{|c|}{ TF K/L } \\
\hline & & & $\beta$ & SE & p Value & $\beta$ & SE & p Value \\
\hline \multirow[t]{4}{*}{ GDF5 } & rs143383 & GOAL & 0.024 & 0.036 & 0.512 & 0.042 & 0.037 & 0.263 \\
\hline & & HCS & 0.103 & 0.055 & 0.064 & 0.077 & 0.058 & 0.180 \\
\hline & & Nottingham & -0.007 & 0.044 & 0.876 & 0.060 & 0.030 & 0.049 \\
\hline & & Summary & 0.033 & 0.045 & 0.463 & 0.058 & 0.021 & 0.006 \\
\hline \multirow[t]{4}{*}{ COG5 } & rs 4730250 & GOAL & -0.006 & 0.054 & 0.908 & 0.001 & 0.056 & 0.988 \\
\hline & & HCS & 0.175 & 0.085 & 0.041 & 0.108 & 0.089 & 0.225 \\
\hline & & Nottingham & 0.021 & 0.047 & 0.657 & 0.016 & 0.037 & 0.655 \\
\hline & & Summary & 0.030 & 0.065 & 0.649 & 0.020 & 0.550 & 0.971 \\
\hline \multirow[t]{4}{*}{ MCF2L } & rs11842874 & GOAL & 0.068 & 0.075 & 0.368 & 0.199 & 0.078 & 0.011 \\
\hline & & HCS & 0.190 & 0.112 & 0.091 & -0.119 & 0.117 & 0.312 \\
\hline & & Nottingham & 0.084 & 0.100 & 0.402 & -0.108 & 0.069 & 0.121 \\
\hline & & Summary & 0.099 & 0.050 & 0.048 & -0.005 & 0.048 & 0.920 \\
\hline
\end{tabular}

GOAL, Genetics of Osteoarthritis and Lifestyle study; HCS, Hertfordshire Cohort Study; Nottingham, Nottingham Case-Control Study. 
A

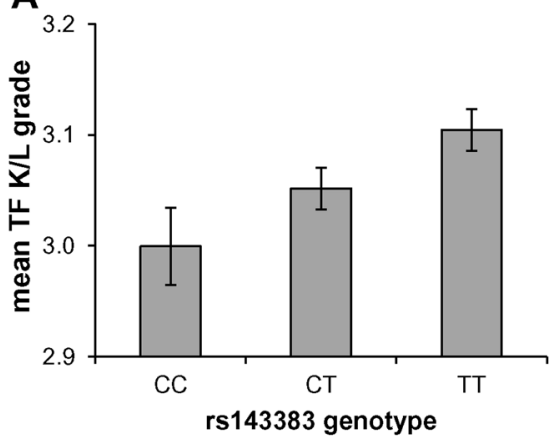

C

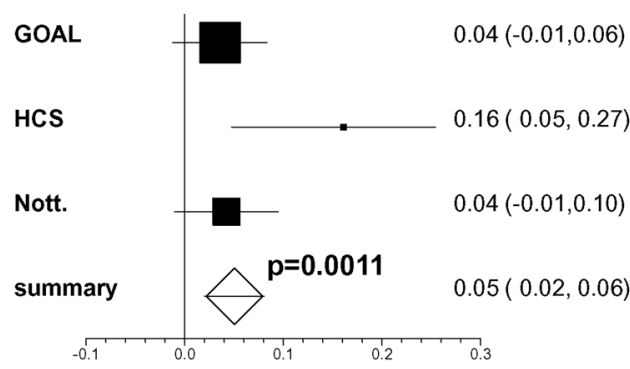

Regression coefficient for association of rs143383 with tibiofemoral K/L grade
B

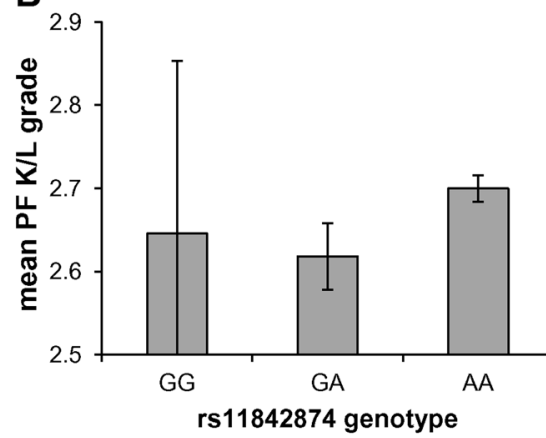

D

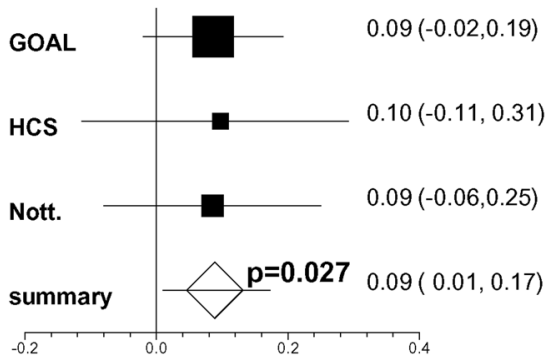

Regression coefficient for association of rs11842874 with patellofemoral K/L grade

$\mathbf{F}$

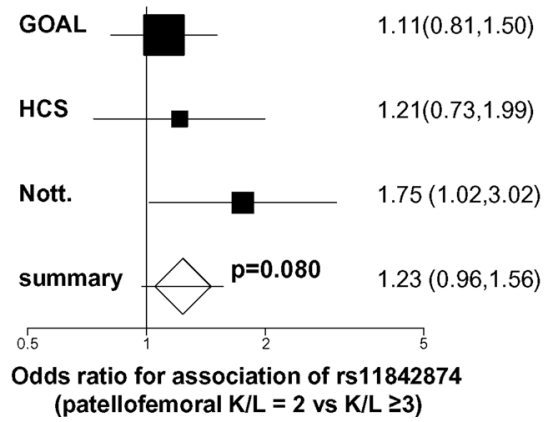

Figure 1 Association between knee K/L grade and gene variants: (A) mean tibiofemoral (TF) K/L grade \pm SE for each GDF5 rs 143388 genotype adjusted for cohort, age, sex and BMI. (B) mean patellofemoral (PF) K/L grade $\pm S E$ or each MCF2L rs11842874 genotype adjusted for cohort, age, sex and body mass index. (C) Forest plot for association between TF K/L and rs143388 for each study cohort, and fixed-effects meta-analysis summary statistics. Only patients with TF K/L $\geq 2$ are included. (D) Forest plot for association between PF K/L and rs 11842874 for each study cohort and fixed-effects meta-analysis summary statistics. Only patients with PF $K / L \geq 2$ are included. (E) Forest plot for association between TF K/L=2 cases versus TF K/L $\geq 3$ and rs 143388 for each study cohort, and fixed-effects meta-analysis summary statistics. (F) Forest plot for association between PF $K / L=2$ cases versus $P F K / L \geq 3$ and rs 11842874 for each study cohort, and fixed-effects meta-analysis summary statistics. All results are adjusted for age, sex and body mass index.

tibiofemoral or patellofemoral compartments. This observation suggests that the mechanisms by which GDF5 influences risk of OA underlie biological processes involved in joint damage, which may be due to an association with disease progression, or this may be due to an influence of genotype on age of onset so that patients who develop the disease earlier in life had more years at risk for progression.

The rs143383 variant maps to the promoter in the GDF5 gene and has been shown to influence expression levels of the gene product, with the predisposing allele $\mathrm{T}$ resulting in lower expression of this chondroprotective growth factor. ${ }^{13}$ On the other hand, the MCF2L gene product has been implicated in peripheral nervous system cell migration as modulated by neurotrophins ${ }^{14}$ and, hence, it has been hypothesised that its role in OA could be via an effect on nociception. The weak correlation found with patellofemoral radiographic grade, suggests that this gene might play a role in joint damage and indicates the need for studies on the functional relevance of this molecule in the biology of joints. No genetic studies have been published specifically on either of the knee compartments, and the association we report with severity does not reach genome-wide significance. However, our results suggest that genetic variation may 
influence specific joint compartments and, given the differences in biomechanics between tibiofemoral and patellofemoral compartments and the integral functioning of the patella within the quadriceps mechanism, this warrants further research into the genetic contribution to both forms of disease. We observed no association with the COG5 SNP. The role of this variant in OA remains unknown although it has been convincingly associated with risk of knee OA. Given the strong linkage disequilibrium in the region it is not possible, genetically, to determine which of the five genes in this gene cluster is the functional one. ${ }^{5}$

We note some study limitations. Our study has only tested samples from the UK; thus, our results may not generalise with respect to other ethnic groups. The association between patellofemoral grade and the MCF2L SNP is only nominally significant $(p=0.027)$ using a linear regression approach, is not significant when severity is analysed as a binary trait, and does not stand a correction for multiple tests. Although potentially of interest, this observation needs to be validated in independent cohort studies.

On the other hand, the association between GDF5 and tibiofemoral grade remains significant after adjustment for multiple tests and, moreover, is statistically significant in the population-based cohort alone (Hertfordshire Cohort Study, $\mathrm{p}<0.006$ ) and in the two case-control cohorts combined ( $p=0.028)$. This association remained significant when a binary definition of severity was used indicating that the lack of normality of the distribution of $\mathrm{K} / \mathrm{L}$ grades was not a statistical artefact. Our data show a continuous effect between gene dosage and, presumably, via the reduced expression of the chondroprotective factor, the extent of joint damage detected by x-ray. In conclusion, we find that GDF5 influences the extent of radiographic damage, but not so the other two genes reported to affect risk of knee OA.

Contributors All authors contributed to the study design, data interpretation and the final manuscript. In addition, AMV analysed and interpreted the data and prepared the manuscript. SD, MW, NA, MD and CC evaluated the study subjects. AMV and MD supervised the study.

Funding This study was supported by the European Commission Framework Programme 7.
Competing interests RAM is an employee and owns stock of Astra Zeneca plc. All other authors declare no conflict of interest.

Patient Consent Obtained.

Provenance and peer review Not commissioned; externally peer reviewed.

\section{REFERENCES}

1. Lopez AD, Mathers CD, Ezzati M, et al. Global and regional burden of disease and risk factors, 2001: systematic analysis of population health data. Lancet 2006;367:1747-57.

2. Chapman $\mathbf{K}$, Valdes AM. Genetic factors in OA pathogenesis. Bone 2011 (In press)

3. Panoutsopoulou K, Southam L, Elliott KS, et al. Insights into the genetic architecture of osteoarthritis from stage 1 of the arcOGEN study. Ann Rheum Dis 2011;70: 864-7.

4. Valdes AM, Evangelou E, Kerkhof HJ, et al. The GDF5 rs143383 polymorphism is associated with osteoarthritis of the knee with genome-wide statistical significance. Ann Rheum Dis 2011;70:873-5.

5. Evangelou E, Valdes AM, Kerkhof HJ, et al. Meta-analysis of genome-wide association studies confirms a susceptibility locus for knee osteoarthritis on chromosome 7q22. Ann Rheum Dis 2011;70:349-55.

6. Day-Williams AG, Southam L, Panoutsopoulou K, et al. A variant in MCF2L is associated with osteoarthritis. Am J Hum Genet 2011;89:446-50.

7. Cornelis FM, Luyten FP, Lories RJ. Functional effects of susceptibility genes in osteoarthritis. Discov Med 2011;12:129-39.

8. Altman RD, Hochberg M, Murphy WA Jr, et al. Atlas of individual radiographic features in osteoarthritis. Osteoarthr Cartil 1995;3 Suppl A:3-70.

9. Lawrence RC, Felson DT, Helmick CG, et al. Estimates of the prevalence of arthritis and other rheumatic conditions in the United States. Part II. Arthritis Rheum 2008;58:26-35

10. Valdes AM, Doherty SA, Zhang W, et al. Inverse relationship between preoperative radiographic severity and postoperative pain in patients with osteoarthritis who have undergone total joint arthroplasty. Semin Arthritis Rheum 2012;41:568-75.

11. Cushnaghan J, Bennett J, Reading I, et al. Long-term outcome following total knee arthroplasty: a controlled longitudinal study. Ann Rheum Dis 2009;68:642-7.

12. Conaghan PG, D'Agostino MA, Le Bars M, et al. Clinical and ultrasonographic predictors of joint replacement for knee osteoarthritis: results from a large, 3-year, prospective EULAR study. Ann Rheum Dis 2010;69:644-7.

13. Miyamoto $\mathbf{Y}$, Mabuchi A, Shi D, et al. A functional polymorphism in the $5^{\prime}$ UTR of GDF5 is associated with susceptibility to osteoarthritis. Nat Genet 2007;39:529-33.

14. Yamauchi J, Chan JR, Miyamoto Y, et al. The neurotrophin-3 receptor TrkC directly phosphorylates and activates the nucleotide exchange factor Dbs to enhance Schwann cell migration. Proc Natl Acad Sci USA 2005;102:5198-203. 